\title{
Domestic Violence among Women in Households in Imo State, Nigeria: Prevalence, Pattern and Determinants
}

\author{
Chukwuma B. Duru ${ }^{1}$, Chinyere M. Aguocha ${ }^{2}$, Anthony C. Iwu ${ }^{1}$, Uche R. Oluoha $^{1}$, Kevin C. Diwe $^{1}$, Ijeoma N. \\ Okedo-Alex ${ }^{3}$, Emmanuel N. Ndukwu ${ }^{4} \&$ Ernest Nwaigbo ${ }^{4}$ \\ ${ }^{1}$ Department of Community Medicine, Imo State University, Owerri, Imo State, Nigeria \\ ${ }^{2}$ Department of Psychiatry, Imo State University, Owerri, Imo State, Nigeria \\ ${ }^{3}$ Department of Community Medicine, Federal Teaching Hospital, Abakaliki, Nigeria \\ ${ }^{4}$ Department of Community Medicine, Imo State University Teaching Hospital, Orlu Imo State, Nigeria \\ Correspondence: Chukwuma B. Duru, Department of Community Medicine, Imo State University, Owerri, Imo \\ State, Nigeria.
}

Received: January 17, 2018

Accepted: February 5, 2018

Online Published: March 2, 2018

doi:10.20849/ajsss.v3i1.323

URL: https://doi.org/10.20849/ajsss.v3i1.323

\begin{abstract}
Introduction: Globally, at least 1 in 3 women has experienced some form of gender based abuse during their life time. Domestic violence occurs across the world in various cultures and affect people across society, irrespective of economic status.
\end{abstract}

Aim: The aim of this study is to assess the prevalence, patterns and determinants of domestic violence among women in households in communities in Imo State.

Methodology: This was a cross-sectional descriptive study carried out among 600 women living in households in Imo State. The multistage sampling technique was used to select the study participants and data was collected using a pretested, interviewer administered and semi-structured questionnaire. The data was analyzed using a computer software (EPI INFO version 7.1.3, CDC, USA), and was presented on frequency tables and percentages. Associations between variables was done using Chi-square and logistic regression where necessary. $P$ value of $\leq 0.05$ was considered statistically significant.

Result: The mean age of women studied was $31.5 \pm 1.4$ years. The prevalence of life time and current experience of domestic violence among the women were $72.8 \%$ and $42.6 \%$ respectively with physical $(49.2 \%)$ and sexual $(31.2 \%)$ abuse being the commonest types experienced. Factors found to be associated with current abuse were; age of women, $(p=0.040)$,religious set attended, $(p=0.004)$, occupational status of women, $(p=0)$, marital status, $(\mathrm{p}=0.028)$, occupational status of partner, $(\mathrm{p}=0)$, place of residence, $(\mathrm{p}=0)$, house hold size, $(\mathrm{p}<0.0001)$, monthly income of women, $(\mathrm{p}<0.0001)$, persons lived with, $(\mathrm{p}=0.002)$ and level of knowledge about domestic abuse, $(\mathrm{p}=0)$.

Conclusion: The prevalence of domestic violence among women in this study was high. Thus, there is need for government at all levels and concerned agencies to do everything within their powers to curb this menace as this will help to improve the lives of our women.

Keywords: domestic violence, prevalence, pattern, determinants, women, households, Imo

\section{Introduction}

Domestic violence has been defined as a pattern of abusive behavior in any relationship that is used by one partner to gain or maintain power and control over another intimate partner (United States Department of Justice, 2017). It is any incident or pattern of incidents of controlling, coercive, threatening behaviour, violence or abuse between those aged 16 or over who are, or have been, intimate partners or family members regardless of gender or sexuality (UK Home Office, 2013). It involves a range of acts designed to make a person subordinate and/or dependent by isolating them from sources of support, exploiting their resources and capacities for personal gain, depriving them of the means needed for independence, resistance and escape and regulating their everyday behavior (UK Home Office, 2013). These are acts or a pattern of acts of assault, threats, humiliation and intimidation or other abuse that are used to harm, punish, or frighten their victim (UK Home Office, 2013). The 
abuse can encompass, but is not limited to psychological, physical, sexual, financial and emotional abuse (UK Home Office, 2013; Intimate Partner Abuse and Relationship Violence Working Group, 2001). And have been associated with injury, mortality and multiple health problems among women (WHO, 2013).

Physical violence includes beating, slapping, throwing objects at the person, pushing, shoving, hitting with fist or objects, kicking, dragging, chocking, burning, using or threatening to use a weapon on the person while sexual violence includes forced sexual intercourse, having sex against your will because of fear about what the partner would do, compelled into engaging in humiliating acts (WHO, 2013). Verbal abuse includes belittling, excessive blaming, insults, humiliation, and threat to take away kids or hurt the person, criticism and intimidation. Financial abuse involves but not limited to withholding allowance/money for various needs including transportation, health and food. Emotional abuse involves trying to isolate the person from family and sources of support. These criteria may vary across studies, regions and settings (WHO, 2013).

Domestic violence occurs in all countries in the world and in all economic strata. Prevalence varies among different regions, countries and communities. (WHO, 2013). It has been found to be higher among the lesser developed countries of the world, especially countries in Africa (WHO, 2013). These variations have been attributed to differences in patriarchal attitude towards women, and tend to be worse in countries where women are viewed as objects of sexual gratification (Arisi and Oromareghake, 2011). These behaviours are used as a weapon to ensure submission (Arisi and Oromareghake, 2011). Another factor is the widespread social acceptance of domestic violence in these societies leading to stigma among the abused and non-report of exposure to abuse (Nigerian Demographic and Health Survey, 2014; Diwe et al, 2016).

Globally about $30 \%$ of all women have experienced domestic abuse with higher rates of physical and sexual abuse (37\%) in Africa, Eastern Mediterranean and South East Asia and lower rates (23\% and 25\%) in high income countries and European and Western Pacific countries respectively (WHO, 2013). Africa has the highest rate and the most severe forms of violence against women globally (WHO, 2005). The rate was seen to be high among young women aged 15-19years and reached a peak among those aged 40-44 years (WHO, 2013).

Studies in Nigeria report the rate of intimate partner violence to range between $13.6 \%$ and $83.4 \%$ (Umeora et al., 2008; Brisibe et al., 2012). These figures differ according to the region of the country studied (Umeora et al., 2008; Brisibe et al., 2012). These rates are considered an underestimation because of the propensity of Nigerian women not to report abuse (Okenwa et al., 2009; Ezechi et al., 2004). Specific rates for physical abuse (46.3\%), economic abuse (21.9\%), sexual abuse (33.8\%) and emotional abuse (39.3\%) have been reported in Nigeria (Ibrahim et al., 2014).

There is a poor level of awareness and high level of justification of domestic violence in Africa and Nigeria in particular (Blideman, 2010; Ilika, 2005; Fawole et al., 2005). Many think it is a husband's right to apply correctional measures on the wife and this is seen even among the abused (Rani et al., 2004). These deep seated beliefs make it difficult and at times impossible for women to report abuse (Ilika, 2005; Diwe et al., 2016; Fawole, 2005) A study carried out in Nigeria found that $66.4 \%$ and $50.4 \%$ of ever-married and single women justified wife beating (Oyediran and Siugo-Abanihe, 2005). Forced sex is condoned and it is considered a cultural and religious norm for a woman to always yield to sexual demand from the husband (Ilika, 2005). Rate of report to law enforcement agencies and divorce as an option to escape from domestic abuse have been found to be culturally unacceptable and largely lead to stigmatization and loss of social support (ilika, 2005; Oluremi, 2015).

Marital status, age at marriage, short duration of marriage, young age, lower literacy status for both spouses, income, and experience of childhood trauma have been associated with Domestic Violence (Bazargan-Hejazi et al, 2013; La Flair et al, 2012).

Thus this study aimed at assessing the pattern, prevalence and determinants of domestic violence among women in households in Imo state, Nigeria.

\section{Methodology}

\subsection{Study Area}

Imo State is one of the 36 States of Nigeria in the South Eastern Region of the country. It has a total population of about 3.93 million people, comprising more males than females (2.03 million and 1.9 million people respectively) and an annual growth rate of about 3.0\%. The majority of the people living in the State are Igbos. The state has one the highest literacy rates in the country which is above $95 \%$. The State is made up of 27 Local Government Areas; 22 are rural while 5 are urban as designated by the National Population Commission (NPC). An urban (Owerri Municipal) and a rural (Oru East) Local Government Area (LGA) were studied (National 
Population Commission, 2006). The major occupations of the people are farming, fishing and artisan jobs in the rural areas while trading and public service are the main occupations among those living in the urban areas. The main religion of the people is Christianity even though few other religions exist in the state.

\subsection{Study Population and Study Design}

This study population comprised women aged 18 years and above living in households in the selected study areas. The study design was a descriptive cross-sectional type.

\subsection{Selection Criteria}

Inclusion criteria: All women 18 years and above whether they were married or not, who have lived in the selected areas of study for at least 6 months prior to the study were enrolled and interviewed.

Exclusion criteria: Women below the age of 18 years, all males irrespective of age, adult females who have not lived up to 6 months in the study areas, visitors from neighboring communities were excluded from the study.

\subsection{Sample Size Determination}

Using the Cochrane sample size formula for cross sectional studies in population above 10,000 people and prevalence of domestic violence in a previous study as stated below;

$$
\mathrm{n}=\mathrm{z}^{2} \mathrm{pq} / \mathrm{d}^{2}(\text { Cochrane, } 1977)
$$

where $\mathrm{n}=$ minimum sample size estimate, $\mathrm{Z}=$ standard normal deviate set at $95 \%$ significance level $(1.96), \mathrm{p}=$ prevalence of domestic physical violence in Nigeria, 28\%( Nigeria Demographic and Health Survey, 2013), $\mathrm{d}=$ the level of precision desired set at 0.05 . Thus substituting the formula above, the minimum sample size required for this study was 323 . But a sample size of 547 was used for this study taking into consideration a likely high attrition rate.

\subsection{Sampling Technique}

The multistage sampling technique was used in this study for the selection of the participants that were enrolled and studied.

Stage one involved the selection of the local government areas that were studied. The Local Government Areas were first stratified into urban and rural LGAs and one LGA was selected from each stratum using simple random sampling by balloting of which Owerri Municipal was selected from the urban while Oru East was selected from the rural. Based on their population size 285 and 262 women were selected from the urban and rural LGA respectively.

Stage two involved the selection of the wards in the LGAs to be studied and using simple random sampling by balloting, five political wards each were selected from both locations.

Stage three involved the selection of Enumeration areas (EAs) from wards that were studied. Two enumeration areas were selected from each ward using simple random sampling by balloting. Thus a total of ten EAs were selected in both locations and about 29 and 26 women per EA respectively were studied in the urban and rural locations of study

Stage four involved the final selection of the respondents and a modified cluster sampling technique was used. Starting from the prominent point in the selected EA, and then moving clockwise, all women in each household who met the criteria was studied until the required sample size was obtained. In each household, only one eligible woman was studied and in households with more than one eligible woman, simple random sampling by balloting was used to select the respondent that was interviewed.

\subsection{Data Collection and Analysis}

Data was collected using a pre-tested, semi structured, interviewer administered questionnaire. The questionnaire contained three sections; Section A: Socio demographic characteristics of respondents, Section B: Awareness and knowledge about domestic violence/abuse, Section C: Prevalence and experience of domestic violence among women. The questionnaires were first translated to Igbo language then back translated to English to ensure that the original meanings of the research questions were maintained. This was to ensure validity and reliability of the study material.

Quantitative data collected were cleaned and validated manually, while a computer software package (EPI INFO version 7.1.3) was used for data entry and analysis. Frequencies and percentages of relevant variables were generated, bivariate and multivariate analysis conducted using Chi-square and logistic regression to test associations between variables. For the purpose of this study, knowledge of respondents about domestic violence 
was scored using five awareness questions, each question assigned scores 0-5 with a maximum total of 25 for all the five questions. The score was graded as poor if the respondent get $0-49 \%$ (of the total of 25 ), fair if the respondent gets 50-69\% of the total and high/good knowledge if the respondents gets $70 \%$ and above of the total score. A p-value $<0.05$ was considered statistically significant.

\subsection{Ethical Consideration}

Ethical approval was gotten from the Department of Community Medicine, Madonna University Nigeria. An informed consent was obtained from each respondent. The study followed the ethical principles guiding the use of human participants in research as declared in Helsinki, 1964.

\section{Results}

Table 1. Sociodemographic and household characteristics of the women

\begin{tabular}{|c|c|c|}
\hline Variables & Frequency $(\mathrm{N}=547)$ & Percentage \\
\hline \multicolumn{3}{|l|}{ Age Group (yrs) } \\
\hline$\leq 20$ & 41 & 7.5 \\
\hline $21-30$ & 243 & 44.4 \\
\hline $31-40$ & 180 & 32.9 \\
\hline $41-50$ & 58 & 10.6 \\
\hline$>50$ & 25 & 4.6 \\
\hline Total & 547 & 100 \\
\hline \multicolumn{3}{|l|}{ Mean $=31.5 \pm 1.4$} \\
\hline \multicolumn{3}{|l|}{ Religion } \\
\hline Catholic & 273 & 50.0 \\
\hline Pentecostal & 226 & 41.3 \\
\hline Orthodox & 38 & 7.0 \\
\hline Traditional & 7 & 1.2 \\
\hline Islam & 3 & 0.5 \\
\hline Total & 547 & 100 \\
\hline \multicolumn{3}{|c|}{ Occupational Status of Women } \\
\hline Employed & 466 & 85.2 \\
\hline Unemployed & 81 & 14.8 \\
\hline Total & 547 & 100 \\
\hline \multicolumn{3}{|l|}{ Marital Status } \\
\hline Currently married & 365 & 66.7 \\
\hline Single & 135 & 24.7 \\
\hline Previously married & 35 & 6.4 \\
\hline Cohabiting & 12 & 2.2 \\
\hline Total & 547 & 100 \\
\hline \multicolumn{3}{|c|}{ Type of Accommodation } \\
\hline Family members alone & 270 & 49.4 \\
\hline Other relatives & 127 & 23.2 \\
\hline Squatting & 59 & 10.8 \\
\hline Living alone & 54 & 9.8 \\
\hline Living with friends & 37 & 6.8 \\
\hline Total & 547 & 100 \\
\hline
\end{tabular}




\begin{tabular}{|c|c|c|}
\hline Employed & 69 & 87.4 \\
\hline Unemployed & 478 & 12.6 \\
\hline Total & 547 & 100 \\
\hline \multicolumn{3}{|c|}{ Number of Children $(\mathrm{N}=365)$} \\
\hline None & 30 & 8.2 \\
\hline $1-5$ & 282 & 77.3 \\
\hline $6-10$ & 53 & 13.7 \\
\hline Total & 365 & 100 \\
\hline Mean $=3.7 \pm 0.5$ & Median =3.6 & \\
\hline \multicolumn{3}{|c|}{ Number of Male Children $(\mathrm{N}=365)$} \\
\hline None & 78 & 21.4 \\
\hline $1-5$ & 244 & 66.8 \\
\hline$>6$ & 43 & 11.8 \\
\hline Total & 365 & 100 \\
\hline \multicolumn{3}{|l|}{ Mean $=2.4 \pm 0.5$} \\
\hline \multicolumn{3}{|c|}{ Number of People In Household } \\
\hline $1-5$ & 198 & 36.2 \\
\hline $6-10$ & 319 & 58.3 \\
\hline $11-15$ & 30 & 5.5 \\
\hline Total & 587 & 100 \\
\hline \multicolumn{3}{|l|}{ Mean $=7.0 \pm 0.3$} \\
\hline \multicolumn{3}{|l|}{ Place of Residence } \\
\hline Rural & 262 & 47.9 \\
\hline Urban & 285 & 52.1 \\
\hline Total & 547 & 100 \\
\hline \multicolumn{3}{|c|}{ How Much Earned in a Month } \\
\hline$<10,000$ & 86 & 15.7 \\
\hline$\approx 10,000-20,000$ & 73 & 13.4 \\
\hline ₹21,000 - 30,000 & 38 & 6.9 \\
\hline$\$ 31,000-50,000$ & 118 & 21.6 \\
\hline$>50,000$ & 232 & 42.4 \\
\hline Total & 547 & 100 \\
\hline \multicolumn{3}{|c|}{ Median $=\$ 37,525 \pm 1881$} \\
\hline \multicolumn{3}{|c|}{ Current Use of Contraception } \\
\hline YES & 123 & 22.5 \\
\hline NO & 424 & 77.5 \\
\hline Total & 547 & 100 \\
\hline
\end{tabular}

\subsection{Socio-Demographic and Household Characteristic of Respondents}

The mean and median ages of the women were $31.5 \pm 1.4$ years and 29.6 years respectively with majority of them being within the ages of 21-40 years of age (77.4\%). Half of the respondents were Catholics (50\%), with majority of them being currently married $(66.7 \%)$ and living alone with their families $(49.4 \%)$ and relatives (23.2\%). Most of the women and their partners were employed; $85.2 \%$ and $87.4 \%$ respectively. The average family size, number of male children, and household size were; $3.7 \pm 0.5,2.4 \pm 0.5$, and $7.0 \pm 0.3$ respectively. The median monthly income of the women was $\$ 37,525 \pm 1881$. 
Table 2. Awareness and knowledge about domestic violence among the women

\begin{tabular}{|c|c|c|}
\hline Variables & Frequency & Percentage \\
\hline \multicolumn{3}{|c|}{ Heard about Domestic Violence $(\mathrm{N}=547$ ) } \\
\hline YES & 440 & 80.4 \\
\hline NO & 107 & 19.6 \\
\hline Total & 547 & 100.0 \\
\hline \multicolumn{3}{|c|}{ **Source of Information about Domestic Violence $(\mathbf{N}=\mathbf{4 4 0})$} \\
\hline Television & 281 & 63.9 \\
\hline Radio & 220 & 50.0 \\
\hline Newspaper & 185 & 42.0 \\
\hline Internet and social media & 156 & 35.5 \\
\hline School & 147 & 33.4 \\
\hline Neighbours & 142 & 32.2 \\
\hline Friends/Relatives & 134 & 30.4 \\
\hline Seminars and workshop & 128 & 27.3 \\
\hline Meetings & 111 & 25.2 \\
\hline Books & 103 & 23.4 \\
\hline Health Personnel & 65 & 14.8 \\
\hline Government Agencies & 63 & 14.3 \\
\hline Non-Governmental Agencies & 55 & 12.5 \\
\hline Posters/Bill Boards & 51 & 11.5 \\
\hline \multicolumn{3}{|c|}{$* *$ Causes of Domestic Violence $(\mathbf{N}=\mathbf{4 4 0})$} \\
\hline Low level of education & 263 & 59.7 \\
\hline Alcohol/Drug abuse & 219 & 49.8 \\
\hline Male ego & 180 & 40.9 \\
\hline Poverty & 158 & 35.9 \\
\hline Behavioral reasons & 152 & 34.5 \\
\hline Young age & 148 & 33.6 \\
\hline Family upbringing & 140 & 31.8 \\
\hline Economic stress & 139 & 31.5 \\
\hline Peer group & 115 & 26.1 \\
\hline \multicolumn{3}{|c|}{ **Forms of Domestic Violence Known (N=440) } \\
\hline Physical abuse & 276 & 62.7 \\
\hline Emotional abuse & 212 & 48.1 \\
\hline Sexual abuse & 206 & 46.8 \\
\hline Verbal abuse & 195 & 44.2 \\
\hline Psychological/Mental abuse & 109 & 24.8 \\
\hline Financial & 63 & 14.3 \\
\hline Spiritual abuse & 49 & 11.1 \\
\hline
\end{tabular}




\begin{tabular}{lll}
\hline$* *$ Relationships Where Domestic Violence Can Occur $\mathbf{~ N ~ = ~ 4 4 0 )}$ & & \\
Marital relationship & 263 & 59.8 \\
Cohabiting & 245 & 55.7 \\
Boyfriend/girlfriend relationship & 196 & 44.5 \\
Casual relationship & 101 & 23.0 \\
Engaged & 92 & 20.9 \\
\hline **Consequences of Domestic Violence among Women $(\mathbf{N}=\mathbf{4 4 0})$ & & \\
Injury and physical harm & 350 & 79.5 \\
Spontaneous abortion & 229 & 52.0 \\
Unwanted Pregnancy & 219 & 49.7 \\
Risks of STIs & 182 & 41.4 \\
Mental problem and suicide & 162 & 36.8 \\
Homicide and other mortality & 94 & 21.4 \\
\hline Level of Knowledge $(\mathbf{N = 5 4 7 )}$ & & \\
Poor (1-49\%) & 337 & 61.6 \\
Fair (50-69\%) & 96 & 17.6 \\
Good $(\geq 70 \%)$ & 114 & 20.8 \\
Total & $\mathbf{5 4 7}$ & $\mathbf{1 0 0}$ \\
\hline
\end{tabular}

\section{$* *=$ Multiple response}

\subsection{Awareness and Knowledge of Domestic Violence/Abuse among the Studied Women}

The commonest cause of domestic violence mentioned by the women was low level of education (59.7\%) while peer group pressure was the least mentioned $(26.1 \%)$. The common forms of domestic violence mentioned were; physical abuse $(62.7 \%)$, emotional abuse $(48.1 \%)$, sexual abuse $(46.8 \%)$ and verbal abuse $(44.2 \%)$. The commonest relationship where domestic violence can occur as mentioned by the women was marital union (59.8\%), this was followed by cohabiting (55.7\%) and living with boyfriend, (44.3\%). Common consequences of domestic violence as mentioned were; physical injuries and harm, (79.5\%), spontaneous abortion, (52.0\%), unwanted pregnancy, (49.7\%), and risk of sexually transmitted infections, (41.1\%). Less than one-third (20.8\%) of the women had good knowledge about domestic violence.

Table 3. Experience of domestic violence among women

\begin{tabular}{lll}
\hline Variable & $\begin{array}{l}\text { Frequency } \\
(\mathbf{N = 5 4 7})\end{array}$ & Percentage \\
\hline Ever Been in an Intimate Relationship & 493 & 90.1 \\
Yes & 54 & 9.9 \\
No & $\mathbf{5 4 7}$ & $\mathbf{1 0 0}$ \\
Total & & \\
\hline Currently in any Kind of Intimate Relationship & 487 & 89.0 \\
Yes & 60 & 11.0 \\
No & $\mathbf{5 4 7}$ & $\mathbf{1 0 0}$ \\
Total & & \\
\hline Ever Experienced Domestic Violence & 398 & 72.8 \\
Yes & 149 & 27.2 \\
No & $\mathbf{5 4 7}$ & $\mathbf{1 0 0}$ \\
Total & & \\
\hline **Type of Abuse Ever Suffered $(\mathbf{N = 3 9 8 )}$ & & \\
\hline
\end{tabular}




\begin{tabular}{|c|c|c|}
\hline Physical abuse & 196 & 49.2 \\
\hline Sexual abuse & 172 & 43.2 \\
\hline Verbal abuse & 132 & 33.2 \\
\hline Emotional abuse & 91 & 22.9 \\
\hline Financial abuse & 62 & 15.6 \\
\hline Spiritual abuse & 47 & 11.8 \\
\hline \multicolumn{3}{|c|}{ **Forms of Physical Abuse Suffered (N=196) } \\
\hline Hitting causing injury & 158 & 80.6 \\
\hline Pushing causing no injury & 115 & 58.7 \\
\hline Pushing causing injury & 80 & 40.8 \\
\hline Slapping or punching causing no injury & 74 & 37.8 \\
\hline Slapping or punching causing injury & 55 & 28.1 \\
\hline Pulling of hair & 25 & 12.8 \\
\hline Attempted strangulation & 11 & 5.6 \\
\hline \multicolumn{3}{|c|}{ **Forms of Verbal Abuse Suffered (N=132) } \\
\hline Name calling & 132 & 100.0 \\
\hline Insults & 123 & 93.1 \\
\hline Blaming & 79 & 59.8 \\
\hline False accusations & 60 & 45.9 \\
\hline Raining curses & 52 & 39.4 \\
\hline \multicolumn{3}{|c|}{ **Forms of Sexual Abuse Suffered $(\mathbf{N}=172)$} \\
\hline Sexual criticisms & 77 & 44.7 \\
\hline Excessive sexual jealousy & 50 & 35.2 \\
\hline Forced to watch pornography & 46 & 26.7 \\
\hline Forced prostitution & 46 & 26.7 \\
\hline Rape & 40 & 23.2 \\
\hline Making sex conditional & 32 & 18.6 \\
\hline \multicolumn{3}{|c|}{ Current Experience of Domestic Violence in the Last 1 Year } \\
\hline Yes & 233 & 42.6 \\
\hline No & 314 & 57.4 \\
\hline Total & 547 & 100 \\
\hline \multicolumn{3}{|l|}{ Frequency of Abuse $(\mathbf{N}=398)$} \\
\hline Once & 28 & 7.0 \\
\hline Twice & 71 & 17.8 \\
\hline Thrice & 52 & 13.1 \\
\hline Greater than thrice & 247 & 62.1 \\
\hline Total & 398 & 100.0 \\
\hline \multicolumn{3}{|l|}{ Ever Reported Any Abuse (N=398) } \\
\hline YES & 368 & 92.5 \\
\hline NO & 30 & 7.5 \\
\hline Total & 398 & 100.0 \\
\hline \multicolumn{3}{|l|}{ **Persons Reported To $(\mathbf{N}=368)$} \\
\hline Family members & 234 & 62.6 \\
\hline Friends & 197 & 53.5 \\
\hline Domestic violence organizations & 116 & 31.6 \\
\hline
\end{tabular}




\begin{tabular}{|c|c|c|}
\hline Police and law enforcement & 10 & 2.6 \\
\hline Church leaders & 10 & 2.6 \\
\hline Social services & 7 & 1.9 \\
\hline School authorities & 5 & 1.3 \\
\hline \multicolumn{3}{|c|}{ Frequency Before Reporting $(\mathrm{N}=368)$} \\
\hline Once & 36 & 9.8 \\
\hline Twice & 62 & 16.8 \\
\hline Thrice & 100 & 27.2 \\
\hline Greater than thrice & 170 & 46.2 \\
\hline Total & 368 & 100.0 \\
\hline \multicolumn{3}{|l|}{ SEX OF ABUSERS (N=398) } \\
\hline Male & 286 & 71.9 \\
\hline Female & 29 & 7.3 \\
\hline Both & 83 & 20.9 \\
\hline Total & 398 & 100 \\
\hline \multicolumn{3}{|l|}{ Status of Abuse (N=398) } \\
\hline Increased & 179 & 45.0 \\
\hline Decreased & 73 & 18.3 \\
\hline Not sure & 70 & 17.6 \\
\hline Stayed the same & 48 & 12.1 \\
\hline Only been abused once & 28 & 7.0 \\
\hline Total & 398 & 100.0 \\
\hline \multicolumn{3}{|c|}{ Living with Abuser or in Close Contact $(\mathrm{N}=398)$} \\
\hline Always & 142 & 35.7 \\
\hline Rarely & 127 & 31.9 \\
\hline Not in contact & 110 & 27.6 \\
\hline Sometimes & 19 & 4.8 \\
\hline Total & 398 & 100.0 \\
\hline \multicolumn{3}{|l|}{ **Reasons for the Abuse $(\mathbf{N}=398)$} \\
\hline Family matters/issues & 228 & 57.3 \\
\hline When we moved in together & 76 & 19.1 \\
\hline When I said I was leaving & 51 & 12.8 \\
\hline After I left & 10 & 16.8 \\
\hline Total & 398 & 100.0 \\
\hline \multicolumn{3}{|c|}{ Any Justification for Domestic Abuse? } \\
\hline Yes & 142 & 26.0 \\
\hline No & 405 & 74.0 \\
\hline Total & 547 & 100.0 \\
\hline \multicolumn{3}{|l|}{ Reasons for Justification ( $\mathrm{N}=142$ ) } \\
\hline Unfaithfulness & 140 & 98.6 \\
\hline Addiction to alcohol or cigarette & 105 & 75.0 \\
\hline Insults & 95 & 68.9 \\
\hline Refusal of sex & 94 & 66.2 \\
\hline Smoking & 90 & 63.4 \\
\hline
\end{tabular}




\subsection{Experience of Domestic Violence/Abuse by the Studied Women}

Most of the women were currently in a relationship, (89.0\%) and have had past relationships, $(90.1 \%)$, with majority (72.8\%) of them having ever experienced any form of domestic violence. The common types ever experienced were; physical abuse, $49.2 \%$, sexual abuse, $43.2 \%$, and verbal abuse (33.2\%). The common forms of physical abuse ever experienced by the respondents were hitting causing injury (80.6\%), pushing causing no injury $(58.7 \%)$, pushing causing injury (40.8\%), and slapping or punching causing no injury $(37.8 \%)$ while the common forms of verbal abuse ever experienced were; name calling (100.0\%), insults (93.1\%), blaming (59.8\%) and false accusations (45.5\%). The commonest sexual abuse ever experienced by the women was sexual criticism (44.7\%), while the least was making sex conditional (18.6\%). About $42.6 \%$ of the women were currently experiencing any form of domestic violence. Majority of the women $(62.1 \%)$, had been abused more than three times in their life time with most of the ever abused women $(92.5 \%)$, reporting the case to someone else commonly after more than three episodes $(46.2 \%)$. The commonest person they report the abuse to was a family member, (62.6\%). A good number of them have experienced an increase in the frequency of abuse (45.0\%) and the commonest reason for the abuse was family issues, $(57.3 \%)$. Majority of the perpetrators were males $(71.9 \%)$, and a good number of the ever abused women (35.7\%) claim that they always live with their abusers. About one-fourth of the women $(26.0 \%)$ agree that that there are cases that domestic abuse can be justified and their commonest reason was partner unfaithfulness, (98.6\%).

Table 4. Determinants of life time experience of domestic violence/abuse among the women

\begin{tabular}{|c|c|c|c|c|c|}
\hline \multirow[t]{2}{*}{ Variables } & \multicolumn{3}{|c|}{ Ever experienced domestic violence/abuse } & \multirow{2}{*}{$\begin{array}{l}\text { Statistics } \\
\left(\mathbf{X}^{2}\right) / \\
\text { p-value }\end{array}$} & \multirow[t]{2}{*}{ OR: $95 \% \mathrm{CI}$} \\
\hline & YES (\%) & NO (\%) & TOTAL (\%) & & \\
\hline \multicolumn{6}{|l|}{ Age Group(Yrs) } \\
\hline$\leq 20$ & $33(80.4)$ & $8(19.5)$ & $41(100)$ & \multirow{6}{*}{$\begin{array}{l}10.744 \\
p=0.001 *\end{array}$} & $0.172(0.029-1.467)$ \\
\hline $21-30$ & $160(65.8)$ & $83(34.2)$ & $243(100)$ & & $0.080 *(0.011-0.604)$ \\
\hline $31-40$ & $137(76.1)$ & $43(23.3)$ & $180(100)$ & & $0.133 *(0.063-0.749)$ \\
\hline $41-50$ & $44(75.9)$ & $14(24.1)$ & $58(100)$ & & $0.132 *(0.006-0.826)$ \\
\hline$\geq 50$ & $24(96.0)$ & $1(4.0)$ & $25(100)$ & & 1.000 \\
\hline Total & $398(72.8)$ & $149(27.2)$ & $547(100)$ & & \\
\hline \multicolumn{6}{|l|}{ Religion } \\
\hline Catholic & $213(78.0)$ & $60(22.0)$ & $273(100)$ & \multirow{5}{*}{$\begin{array}{l}0.189 \\
p=0.663\end{array}$} & 1.000 \\
\hline Pentecostal & $145(64.2)$ & $81(35.8)$ & $226(100)$ & & $0.504 *(0.040-0.749)$ \\
\hline Orthodox & $32(84.2)$ & $6(15.8)$ & $38(100)$ & & $1.502(0.600-3.762)$ \\
\hline Others $^{1}$ & $8(80.0)$ & $2(20.0)$ & $10(100)$ & & $1.127(0.233-5.447)$ \\
\hline Total & $398(72.8)$ & $149(27.2)$ & $547(100)$ & & \\
\hline \multicolumn{6}{|c|}{ Occupational Status of Women } \\
\hline Civil servant & $331(71.0)$ & $135(29.0)$ & $466(100)$ & \multirow{3}{*}{$\begin{array}{l}4.755 \\
p=0.029 *\end{array}$} & 1.000 \\
\hline Unemployed & $67(82.7)$ & $14(17.3)$ & $81(100)$ & & $1.952 *(1.061-3.851)$ \\
\hline Total & 398 (72.8) & $149(27.2)$ & $547(100)$ & & \\
\hline \multicolumn{6}{|l|}{ Marital Status } \\
\hline Currently Married & $245(67.1)$ & $120(32.9)$ & $365(100)$ & \multirow{5}{*}{$\begin{array}{l}15.642 \\
\mathbf{P}<0.0001^{*}\end{array}$} & 1.000 \\
\hline Single & $115(85.2)$ & $20(14.8)$ & $135(100)$ & & $2.816 *(1.670-4.750)$ \\
\hline Previously married & $29(82.9)$ & $6(17.1)$ & $35(100)$ & & $*$ \\
\hline Cohabiting & $9(75.0)$ & $3(25.0)$ & $12(100)$ & & $2.367(0.930-7.152)$ \\
\hline Total & $398(72.8)$ & $149(27.2)$ & $547(100)$ & & $1.469(0.358-8.582)$ \\
\hline
\end{tabular}




\begin{tabular}{|c|c|c|c|c|c|}
\hline \multicolumn{6}{|l|}{ Persons Lived with } \\
\hline Family members alone & $200(74.1)$ & $70(25.9)$ & $270(100)$ & \multirow{6}{*}{$\begin{array}{l}3.888 \\
p=0.049 *\end{array}$} & 1.000 \\
\hline Other relatives & $107(84.3)$ & $20(15.7)$ & $127(100)$ & & $1.872 *(1.081-3.244)$ \\
\hline Squatting & $40(67.8)$ & $19(32.2)$ & $59(100)$ & & $0.737(0.400-1.356)$ \\
\hline Living alone & $33(61.1)$ & $21(38.9)$ & $54(100)$ & & $0.550(0.299-1.028)$ \\
\hline Living with friends & $18(48.6)$ & $19(51.4)$ & $37(100)$ & & $0.332 *(0.165-0.668)$ \\
\hline Total & $398(72.8)$ & $149(27.2)$ & $547(100)$ & & \\
\hline \multicolumn{6}{|c|}{ Occupational Status of Partner } \\
\hline Employed & $341(71.9)$ & $137(28.1)$ & $478(100)$ & \multirow{3}{*}{$\begin{array}{l}1.917 \\
p=0.167\end{array}$} & 1.000 \\
\hline Unemployed & $57(82.6)$ & $15(17.4)$ & $69(100)$ & & $1.527(0.836-2.788)$ \\
\hline Total & 398 (72.8) & $149(27.2)$ & $547(100)$ & & \\
\hline \multicolumn{6}{|l|}{ Family Size(N=365) } \\
\hline No Child & $14(46.7)$ & $16(53.3)$ & $30(100)$ & \multirow{4}{*}{$\begin{array}{l}6.198 \\
p=0.013 *\end{array}$} & 1.000 \\
\hline $1-5$ & $193(68.4)$ & $89(31.6)$ & $282(100)$ & & $2.478 *(1.159-5.299)$ \\
\hline$>6$ & $38(71.7)$ & $15(28.3)$ & $53(100)$ & & $2.895 *(1.138-7.365)$ \\
\hline Total & $245(67.1)$ & $120(32.9)$ & $365(100)$ & & \\
\hline \multicolumn{6}{|l|}{ Place of Residence } \\
\hline Rural & $133(49.2)$ & $129(50.8)$ & $262(100)$ & \multirow{3}{*}{$\begin{array}{l}122.767 \\
p=0 *\end{array}$} & 1.000 \\
\hline Urban & $265(93.0)$ & $20(7.0)$ & $285(100)$ & & $12.851 *(7.68-21.51)$ \\
\hline Total & $398(72.8)$ & $149(27.2)$ & $557(100)$ & & \\
\hline \multicolumn{6}{|l|}{ Household Size } \\
\hline $1-5$ & $174(87.9)$ & $24(12.1)$ & $198(100)$ & \multirow{4}{*}{$\begin{array}{l}22.851 \\
\mathbf{P}<0.0001 *\end{array}$} & 1.000 \\
\hline $6-10$ & $201(63.0)$ & $118(37.0)$ & $319(100)$ & & $0.235 *(0.145-0.381)$ \\
\hline $11-15$ & $23(76.7)$ & $7(23.3)$ & $30(100)$ & & $0.453(0.176-1.169)$ \\
\hline Total & $398(72.8)$ & $149(27.2)$ & $547(100)$ & & \\
\hline \multicolumn{6}{|c|}{ Number of Male Children $(\mathrm{N}=365)$} \\
\hline None & $69(88.5)$ & $9(11.5)$ & $78(100)$ & \multirow{4}{*}{$\begin{array}{l}20.467 \\
P<0.0001 *\end{array}$} & 1.000 \\
\hline $1-5$ & $165(67.6)$ & $79(32.4)$ & $244(100)$ & & $0.272 *(0.129-0.574)$ \\
\hline$>6$ & $11(25.6)$ & $32(74.4)$ & $43(100)$ & & $0.045 *(0.017-0.119)$ \\
\hline Total & $245(67.1)$ & $120(32.9)$ & $365(100)$ & & \\
\hline \multicolumn{6}{|c|}{ Current Use of Contraception } \\
\hline Yes & $99(80.5)$ & $24(19.5)$ & $123(100)$ & \multirow{3}{*}{$\begin{array}{l}4.781 \\
p=0.029 *\end{array}$} & 1.000 \\
\hline No & $299(70.5)$ & $125(29.5)$ & $424(100)$ & & $1.725 *(1.054-2.822)$ \\
\hline Total & $398(72.8)$ & $149(27.2)$ & $547(100)$ & & \\
\hline \multicolumn{6}{|l|}{ Monthly Income } \\
\hline$<\mathrm{N} 10,000$ & $82(95.3)$ & $4(4.7)$ & $86(100)$ & \multirow{4}{*}{$\begin{array}{l}28.689 \\
p<0.0001 *\end{array}$} & 1.000 \\
\hline $\mathrm{N} 10,000-\mathrm{N} 50,000$ & $200(87.3)$ & $29(12.7)$ & $229(100)$ & & $0.336 *(0.115-0.987)$ \\
\hline$>\mathrm{N} 50,000$ & $116(50.0))$ & $116(50.0)$ & $232(100)$ & & $0.049 *(0.017-0.138)$ \\
\hline Total & $398(72.8)$ & $149(27.2)$ & $547(100)$ & & \\
\hline \multicolumn{6}{|l|}{ Knowledge level } \\
\hline Poor $(0-49 \%)$ & $275(81.6)$ & $62(18.4)$ & $337(100)$ & \multirow{4}{*}{$\begin{array}{l}24.925 \\
p<0.001 *\end{array}$} & 1.000 \\
\hline Fair $(50-69 \%)$ & $73(76.0)$ & $23(24.0)$ & $96(100)$ & & $0.716(0.416-1.232)$ \\
\hline Good knowledge ( $\geq 70 \%$ ) & $56(49.1)$ & $58(50.9)$ & $114(100)$ & & $0.218 *(0.138-0.345)$ \\
\hline Total & $398(72.8)$ & $149(27.2)$ & $547(100)$ & & \\
\hline
\end{tabular}

Others $^{1}=$ Traditional religion and Islam 


\subsection{Determinants of Life Time Experience of Domestic Violence/Abuse among Respondents}

Factors found to be associated with life time experience of domestic violence in this study were; age of women, $\left(X^{2}=10.744, p=0.001\right)$, occupational status of women, $\left(\left(X^{2}=4.755, p=0.029\right)\right.$, marital status, $\left(X^{2}=15.642\right.$, $\mathrm{p}<0.0001)$, persons lived with, $\left(X^{2}=3.888, p=0.049\right)$, family size, $\left(X^{2}=6.198, p=0.013\right)$, place of residence, $\left(X^{2}=\right.$ $122.769, p=0)$, household size, $\left(X^{2}=22.851, p<0.0001\right)$, number of male children, $\left(X^{2}=20.467, p<0.0001\right)$, current use of contraception, $\left(X^{2}=4.781, p=0.028\right)$, monthly income of women, $\left(X^{2}=28.689, p<0.0001\right)$, and level of knowledge about domestic violence, $\left(\mathrm{X}^{2}=24.925, \mathrm{p}<0.0001\right)$. Women in the younger age group, 21-30 years of age, (OR:0.080; 0.011-0.604), who attends Pentecostal Christian denomination, (OR:0.504; 0.040-0.749), living with friends, (OR:0.332; 0.165-0.668), staying in household of about 6-10 people, (OR:0.235; 0.145-0.381), had more than six male children, (OR: 0.045; 0.017-0.119), earn more than 50,000 naira, (OR:0.049, 0.017-0.138), with a high level of knowledge about domestic violence/abuse, (OR: 0.218; 0.138-0.345), were less likely to have ever experienced any form of domestic violence. On the other hand, older women above 50 years of age who were unemployed, (OR: 1.952; 1.061-3.851), single, (OR: 2.818; 1.670-4.750), living with other relatives, (OR: 1.872; 1.081-3.244), having a family size greater than six people, (OR:2.895; 1.138-7.365), living in an urban area, (OR:12.851;7.68-12.51 and currently using any form contraception, (OR: $1.725 ; 1.054-2.822$ ) were more likely to have ever experienced any form of domestic abuse than their counterparts.

Table 5. Determinants of current experience of domestic violence/abuse among the women

\begin{tabular}{|c|c|c|c|c|c|}
\hline \multirow[t]{2}{*}{ Variables } & \multicolumn{3}{|c|}{ Currently Expriencing Domestic Violence } & \multirow{2}{*}{$\begin{array}{l}\text { Statistics } \\
\left(\mathbf{X}^{2}\right) / \mathbf{p} \text {-value }\end{array}$} & \multirow[t]{2}{*}{ Or: $95 \%$ CI } \\
\hline & Yes $(\%)$ & No $(\%)$ & Total $(\%)$ & & \\
\hline \multicolumn{6}{|l|}{ Age Group(Yrs) } \\
\hline$\leq 20$ & $5(12.2)$ & $36(87.8)$ & $41(100)$ & & 1.000 \\
\hline $21-30$ & $54(22.2)$ & 189 (77.8) & $243(100)$ & 4.208 & $2.057(0.770-5.498)$ \\
\hline $31-40$ & $136(75.6)$ & $44(24.4)$ & $180(100)$ & $\mathrm{p}=0.040 *$ & $22.25 *(8.230-60.210)$ \\
\hline $41-50$ & $28(48.3)$ & $30(51.7)$ & $58(100)$ & & $6.720 *(2.310-19.549)$ \\
\hline$>50$ & $10(40.0)$ & $15(50.0)$ & $25(100)$ & & $4.800 *(1.401-16.438)$ \\
\hline Total & $233(42.6)$ & 314 (57.4) & $547(100)$ & & \\
\hline \multicolumn{6}{|l|}{ Religion } \\
\hline Catholic & $125(45.8)$ & $148(54.2)$ & $273(100)$ & & 1.000 \\
\hline Pentecostal & $74(32.7)$ & $152(67.3)$ & $226(100)$ & 8.176 & $0.576 *(0.400-0.831)$ \\
\hline Orthodox & $28(73.7)$ & $10(26.2)$ & $38(100)$ & $\mathrm{p}=0.004 *$ & $3.315 *(1.550-7.091)$ \\
\hline Others $^{1}$ & $6(60.0)$ & $4(40.0)$ & $10(100)$ & & $1.776(0.490-6.435)$ \\
\hline Total & $233(42.6)$ & 314 (57.4) & $547(100)$ & & \\
\hline \multicolumn{6}{|l|}{ Occupational Status } \\
\hline Employed & $166(35.6)$ & $300(64.4)$ & $466(100$ & 62.589 & 1.000 \\
\hline Unemployed & $67(82.7)$ & $14(17.3)$ & $81(100)$ & $\mathbf{p}=\mathbf{0}^{*}$ & $8.649 *(4.717-15.860)$ \\
\hline Total & $233(42.6)$ & $314(57.4)$ & $547(100)$ & & \\
\hline \multicolumn{6}{|l|}{ Marital Status } \\
\hline Currently Married & $143(39.2)$ & $222(60.8)$ & $365(100)$ & & 1.000 \\
\hline Single & $68(50.4)$ & 67 (49.6) & $135(100)$ & 4.822 & $1.576 *(1.059-2.345)$ \\
\hline previously married & $13(37.2)$ & $22(62.9)$ & $35(100)$ & $\mathrm{p}=0.028^{*}$ & $0.917(0.436-1.879)$ \\
\hline Cohabiting & $9(75.0)$ & $3(25.0)$ & $12(100)$ & & $4.657 *(1.240-17.495)$ \\
\hline Total & $233(42.6)$ & $314(57.4)$ & $547(100)$ & & \\
\hline \multicolumn{6}{|l|}{ Persons Lived With } \\
\hline Family members alone & 105 (38.9) & $165(61.1)$ & $270(100)$ & & 1.000 \\
\hline Other relatives & $73(57.5)$ & $54(42.5)$ & $127(100)$ & 10.303 & $2.124 *(1.384-3.261)$ \\
\hline Squatting & $40(67.8)$ & $19(32.2)$ & $59(100)$ & $\mathrm{p}=0.002 *$ & $3.308 *(1.819-6.019)$ \\
\hline
\end{tabular}




\begin{tabular}{|c|c|c|c|c|c|}
\hline Living alone & $5(9.3)$ & $49(90.7)$ & $54(100)$ & & $0.160 *(0.062-0.416)$ \\
\hline Living with friends & $10(27.0)$ & $27(73.0)$ & $37(100)$ & & $0.582(0.271-1.252)$ \\
\hline Total & $233(42.6)$ & $314(57.4)$ & $547(100)$ & & \\
\hline \multicolumn{6}{|c|}{ Occupational Status of Partner } \\
\hline Employed & $176(36.8)$ & $302(63.2)$ & $478(100)$ & 51.700 & 1.000 \\
\hline Unemployed & $57(87.6)$ & $12(17.4)$ & $69(100)$ & $\mathbf{p}=0 *$ & $8.151 *(4.256-15.608)$ \\
\hline Total & $233(42.6)$ & $314(57.4)$ & $547(100)$ & & \\
\hline \multicolumn{6}{|l|}{ Family Size (N=365) } \\
\hline No child & $14(46.7)$ & $16(53.3)$ & $30(100)$ & & 1.000 \\
\hline $1-5$ & $109(38.7)$ & $173(61.3)$ & $282(100)$ & 0.177 & $0.720(0.338-1.543)$ \\
\hline$>6$ & $20(37.3)$ & $33(62.3)$ & $53(100)$ & $\mathrm{p}=0.674$ & $0.693(0.280-1.716)$ \\
\hline Total & $143(39.2)$ & $222(60.8)$ & $365(100)$ & & \\
\hline \multicolumn{6}{|l|}{ Place of Residence } \\
\hline Rural & $63(24.0)$ & $199(76.0)$ & $262(100.0)$ & 70.767 & \\
\hline Urban & $170(59.6)$ & $115(40.4)$ & $285(100.0)$ & $\mathbf{p}=0^{*}$ & $4.669 *(3.228-6.755)$ \\
\hline Total & 233 (42.6) & $314(57.4)$ & $547(100.0)$ & & \\
\hline \multicolumn{6}{|l|}{ Household Size } \\
\hline $1-5$ & $43(21.7)$ & $155(78.3)$ & $198(100.0)$ & & 1.000 \\
\hline 6-10 & $174(54.5)$ & $145(45.5)$ & $319(100.0)$ & 19.560 & $4.326 *(2.890-6.475)$ \\
\hline $11-15$ & $16(53.3)$ & $14(46.7)$ & $30(100.0)$ & $\mathrm{p}<0.0001 *$ & $4.120 *(1.864-9.103)$ \\
\hline Total & $233(42.6)$ & $314(57.4)$ & $547(100.0)$ & & \\
\hline \multicolumn{6}{|c|}{ Number of Male Children $(\mathrm{N}=365)$} \\
\hline No male child & $36(46.2)$ & $42(53.8)$ & $78(100.0)$ & & 1.000 \\
\hline $1-5$ & 99 (40.6) & $145(59.4)$ & $244(100.0)$ & 1.226 & $0.797(0.477-1.331)$ \\
\hline$>6$ & $8(18.6)$ & $35(81.4)$ & $43(100.0)$ & $\mathrm{P}=0.269$ & $0.267 *(0.110-0.648)$ \\
\hline Total & $143(39.2)$ & $222(60.8)$ & $365(100.0)$ & & \\
\hline \multicolumn{6}{|c|}{ Current Use of Contraception } \\
\hline Yes & $59(47.0)$ & $64(52.0)$ & $123(100.0)$ & 1.872 & \\
\hline No & $174(41.0)$ & $250(59.0)$ & $424(100.0)$ & $\mathrm{p}=0.171$ & $0.755(0.545-1.130)$ \\
\hline Total & $233(42.6)$ & $314(57.4)$ & $547(100.0)$ & & \\
\hline \multicolumn{6}{|l|}{ Month Income } \\
\hline $\mathrm{N} 10,000$ & $55(64.0)$ & $31(32.9)$ & $86(100.0)$ & & 1.000 \\
\hline $\mathrm{N} 10,00-\mathrm{N} 50,000$ & $104(41.4)$ & $125(54.6)$ & $229(100.0)$ & 19.670 & $0.469 *(0.281-0.782)$ \\
\hline$>\mathrm{N} 50,000$ & $74(31.9)$ & $158(68.1)$ & $232(100.0)$ & $\mathbf{p}<0.0001 *$ & $0.264 *(0.157-0.444)$ \\
\hline Total & $233(42.6)$ & $314(57.4)$ & $547(100.0)$ & & \\
\hline \multicolumn{6}{|l|}{ Knowledge Level } \\
\hline Poor $(0-49 \%)$ & $203(60.2)$ & $134(39.8)$ & $337(100)$ & & 1.000 \\
\hline Fair (50-69\%) & $14(14.6)$ & $82(85.4)$ & $96(100)$ & 111.159 & $0.113 *(0.061-0.207)$ \\
\hline Good $(\geq 70 \%)$ & $16(14.0)$ & $98(86.0)$ & $114(100)$ & $\mathbf{p}=0^{*}$ & $0.108 *(0.061-0.370)$ \\
\hline Total & $233(58.5)$ & $165(41.5)$ & $547(100)$ & & \\
\hline
\end{tabular}

Others $^{1}=$ Traditional religion and Islam, $*$ = statistically significant

3.5 Determinants of Current Domestic Violence/Abuse against the Studied Women

Age of the women was found to significantly influence the occurrence of domestic violence/abuse, $\left(\mathrm{X}^{2}=4.208\right.$, $\mathrm{p}=0.040$ ) with women within the $31-40$ years age bracket (OR: $22.25 ; 8.230-60.210$ ) being the most likely to be 
currently experiencing domestic violence/abuse. Religious sect attended by the women influenced the current occurrence of domestic violence/abuse significantly, $\left(X^{2}=8.176, p=0.004\right)$ with women in orthodox denominations having the highest likelihood of being currently abused, (OR: 3.315; 1.556-7.091) while their counterparts in Pentecostal denominations had the least likelihood of being currently abused, (OR: 0.576, 0.400-0.831). Occupational status of women $\left(X^{2}=62.509, p=0\right)$ and their partners, $\left(X^{2}=51.700, p=0\right)$ was associated significantly with experiencing current abuse with unemployed women, (OR: 8.469; 4.417-15.860) and those with unemployed partners, (OR: 8.151; 4.256-15.608) being the most likely to be abused. Women cohabiting with their partners were about 5 times more likely to have been currently abused when compared to their currently married counterparts, (OR: 4.657; 1.240-17.4950). Women squatting with someone, (OR: 3.308; 1.819-6.019) were more likely to have been abused currently while those living alone (OR: $0.160 ; 0.062-0.416)$ were the least likely to have been currently abused. Place of residence of the women, $\left(X^{2}=70.767, p=0\right)$ and household size, $\left(X^{2}=19.560, p<0001\right)$ was found to significantly influence current occurrence of domestic violence/abuse with urban women being about 5 times more likely to abused than their rural counterparts, (4.669; 3.228-6.755) and women living in larger households (6-10) being more likely the be currently abused, (OR: $4.326 ; 2.890-6.475)$. Also women with male children $>6$ in number were the least likely to be currently abused, (OR: $0.267 ; 0.110-0.648)$. Experience of current domestic abuse/violence was found to significantly reduce with increasing monthly income of the women, $\left(X^{2}=19.670, p<0001\right)$ with women who earn greater than 50,000 naira monthly being the least likely to have been currently abused (OR: 0.264; 0.157-0.444). Also this was the trend when level of knowledge about domestic violence and occurrence of domestic abuse was assessed, $\left(\left(\mathrm{X}^{2}=111.159\right.\right.$, $\mathrm{p}=0$ ) with women that have good knowledge being the least likely to have been currently abused, (OR: 0.108 ; 0.061-0.370).

\section{Discussion}

This study found a high rate of domestic violence, $72.8 \%$ and $42.6 \%$ being life time and current rates respectively. This is much higher than the reported global rates and rates reported in Africa but falls within the range reported in Nigeria (WHO, 2013; Umeora et al., 2008; Brisibe et al., 2012). The high rate reported in this study can be attributed to socio-cultural factors. There are very strong patriarchal attitudes in Nigeria. Women are regarded as no better than chattels (Ekenta et al., 2012). These patriarchal structures were developed to commandeer women into existing power structures where they had to submit to their partners (Ritzer, 1996). It is culturally accepted for a man to punish his wife and the society turns a blind eye to it (Bradley, 1994) though this trend is gradually changing. The country is deeply religious and most of the religious sects preach that women should obey their husbands and that their husbands should in turn love them, this may have been misunderstood by many of our men who feel that women should be subservient to them. This could lead to a sense of entitlement among the men. This could account for the very high rates of experience of domestic violence among the members of the different denominations found in this study. The rate was highest among the members of non-Catholic Orthodox sects and lowest among the Pentecostal denominations. This is in keeping with studies that have reported greater abuse among other orthodox sects (Feseha et al., 2012).

This study found a low rate of justification (26.0\%) for domestic violence among the women. The rate was lower than 50.4\%-75\% reported in other studies within and outside Nigeria (Fawole et al., 2005; Lawoko, 2006; Ilika, 2005; Oyediran and Siugo-Abanihe, 2005). The lower justification rate found in this study may explain the higher rate of help seeking found (92.5\%) compared to 37.6\% previously reported (Shrivastava and Shrivastava, 2013). It was found that most of the respondents reported to family members who most time take no concrete action to end abuse but merely convince the women to accept abuse as a norm. Very few of those who had experienced abuse reported to the social services, the law enforcement and the church. This is similar to what has been previously reported (Ilika, 2005; Yusuf, 2000). This has been attributed to lack of support from the families who would rather have family issues kept behind closed doors, poor response and victim blaming by law enforcement (Ilika, 2005; Abayomi and Olabode, 2013).

This study found domestic abuse to be increased among the young and middle aged, single, unemployed, low income, urban dwellers and those from large households. This is similar to what has been reported in other studies (Srinivasan and Bedi, 2007; CDC, 2006). Some studies also reported that higher levels of education and economic status of women to be protective against women's risk of domestic violence (Koenig et al., 2006; Hoffman et al, 1996). This set of women may not have the necessary exposure and financial empowerment to challenge their abusers, who may be providing for their daily needs. Physical abuse was the commonest form of abuse reported in this study. This is similar to what has been reported in other studies (Bazargan-Hejazi et al., 2013; Ibrahim et al., 2014). This may be due to the dramatic nature of physical abuse. 


\section{Conclusion}

In conclusion the prevalence of domestic violence against women was high in this study, still prevalent in our today's society and it was found to be worse among single women with poor knowledge about domestic violence and who were likely of low socioeconomic status. This has grave public health consequences if not properly tackled. Thus there is need for government at all levels and all relevant agencies to create awareness on the consequences of domestic violence against women and also implement already existing policies geared towards its prevention and punishment of perpetrators. There is also need to educate our women and empower them so that they can be financially independent.

Domestic violence occurs globally and its occurrence is widely under-reported especially in third world countries like Nigeria where the society seems to turn a deaf ear to this menace. This problem is caused by a combination of cultural and religious influences which view women as lesser humans when compared to their male counterparts. The findings of this study revealed the occurrence of domestic violence and factors influencing it in this part of the world. Data generated from this study will strengthen existing baseline information on domestic violence in the state which may help in the development of policies that is geared towards the control and prevention of domestic violence and its consequences in the state. This study is descriptive and exploratory in nature so care should be taken in generalizing its findings to the entire population. The researchers suggest the need to conduct a more extensive research on this topic in this part of the world.

\section{Acknowledgement}

We thank all the participants in this study and the research assistants who helped during the data collection.

Authors' Contributions: All the authors participated in the study.

Competing Interest: The authors hereby declare that there are no competing interests.

Source of Funding: There was no external source of funding

\section{References}

Abayomi, A. A, \& Olabode, K. T. (2013). Domestic Violence and Death: Women as Endangered Gender in Nigeria. American Journal of Sociological Research, 3(3), 53-60.

Arisi, R. O., \& Oromareghake, P. (2011). Cultural Violence and the Nigerian Woman. African Research Review, 5(4), 369-381. https://doi.org/10.4314/afrrev.v5i4.69290

Bazargan-Hejazi, S., Medeiros, S., Mohammadi, R., Lin, J., \& Dalal, K. (2013). Patterns of Intimate Partner Violence: A study of female victims in Malawi. $J$ Inj Violence Res, 5(1), 38-50. https://doi.org/10.5249/jivr.v5i1.139

Blideman, A. (2010). Nursing students' attitudes towards domestic violence: A quantitative study at Kilimanjaro Christian Medical College, Moshi, Tanzania. Red Cross University College. (Thesis).

Bradley, C. (1994). Why male violence against women is a development issue: Reflections from Paupau, New Guinea In Davies M. (Ed.), Women and violence: Realities and responses worldwide. New York: Zed Books.

Brisibe, S., Ordinioha, B., \& Dienye, P. O. (2012). Intersection between alcohol abuse and intimate partner's violence in a rural Ijaw community in Bayelsa State, South-South Nigeria. J Interpers Violence, 27, 513-22. https://doi.org/10.1177/0886260511421676

Center for Disease Control \& Prevention, (2006). Intimate partner violence-fact sheet CDC Washington. Retrieved September 21, 2017, from at www.cdc.gov/injury

Cochrane, W. (1977). Sampling techniques $\left(3^{\text {rd }}\right.$ ed.). New York: John Willey and sons.

Diwem, K. C., Duru, C. B., Aguocha, C., Uwakwe, K. A., Merenu, I. A., Emerole, C.O., \& Iwu, A.C. (2016). Prevalence, pattern and determinants of Intimate Partner Violence among Single Females in Tertiary Institutions in Imo State, Nigeria. International Journal of Research and Review, 3(6), 26-35.

Ekenta, C. M., Mohammed, A. B., \& Afolabim K. O. (2012). Gender analysis of land ownership structures and agricultural production in Imo state, Nigeria. Journal of Economics and Sustainable Development, 3(9), 855. Retrived September 20, 2017, from www.iiste.org

Ezechi, O. C., Kalu, B. K., Ezechi, L. O., Nwokoro, C. A., Ndububa, V. I., \& Okeke, G. C. (2004). Prevalence and pattern of domestic violence against pregnant Nigerian women. Journal of Obstetrics and Gynaecology, 24(6), 652-656. https://doi.org/10.1080/01443610400007901 
Fawole, O. I., Aderonmu, A. L., \& Fawole, A. O. (2005). Intimate partner abuse: wife beating among civil servants in Ibadan, Nigeria. Afr J Reprod Health, 9(2), 54-64. https://doi.org/10.2307/3583462

Feseha, G., Mariam, A., \& Gerbaba, M. (2012). Intimate partner physical violence among women in Shimelba refugee camp, northern Ethiopia. BMC Public Health, 12, 125. https://doi.org/10.1186/1471-2458-12-125

Hoffman, K., Demo, D. H., \& Edwards, J. N. (1996). Physical wife abuse in a non-western society: an integrated theoretical approach. J Marriage Fam, 56, 131-146. https://doi.org/10.2307/352709

Ibrahim, M. S., Bashir, S. S., Umar, A. A., Gobir, A. A., \& Idris, S. H. (2014). Men's perspectives on intimate partner abuse in an urban community in North-Western Nigeria. Ann Nigerian Med, 8, 37-41. https://doi.org/10.4103/0331-3131.141028

Ilika, A. L. (2005). Women's perceptions of partner violence in a rural Igbo community. Africa Journal of Reproductive Health, 9, 77-88. https://doi.org/10.2307/3583414

Intimate Partner Abuse and Relationship Violence Working Group. (2001). Intimate Partner Abuse and Relationship Violence. Paper presented at the annual convention of the American Psychological Association in San Francisco, August, 2001. Retrieved September 21, 2017, from https://www.apa.org

Koenig, M. A., Stephenson, S., Ahmed, S., Jejeebhoy, S. J., \& Campbell, J. (2006). individual and contextual determinants of domestic violence in North India. Am J Public Health, 96(1), 132-138. https://doi.org/10.2105/AJPH.2004.050872

La Flair, L. N., Bradshaw, C. P., \& Campbell, J. C. (2012). Intimate partner violence/abuse and depressive symptoms among female healthcare workers: Longitudinal findings. Women's Health Issues, 22(1), 53-59. https://doi.org/10.1016/j.whi.2011.07.001

Lawoko, S. (2006). Factors associated with attitudes towards violence: a study of women in Zambia. Violence and Victims, 21, 645-656. https://doi.org/10.1891/0886-6708.21.5.645

National Population Commission (NPC) [Nigeria] and ICF International. (2014). Nigeria Demographic and Health Survey 2013. Abuja, Nigeria, and Rockville, Maryland, USA: NPC and ICF International.

National Population Commission (NPC), Federal Republic of Nigeria. (2006). 2006 Population and Housing Census; Priority Table III. Retrieved September 21, 2017, from http://www.population.gov.ng/images/Vol\%2003\%20Table\%20DSx\%20LGAPop\%20by\%20SDistrict-PD F.pdf

Okenwa, L., Lawoko, S., \& Jansson, B. (2009). Factors associated with disclosure of intimate partner violence among Women in Lagos, Nigeria. J Inj Violence Res, 1(1), 37-47. https://doi.org/10.5249/jivr.v1i1.15

Oluremi, F. D. (2015). Domestic violence against women In Nigeria. European Journal of Psychological Research, 2(1), 24.

Oyediran, K. A., \& siugo-Abanihe, U. (2005). Perceptions of Nigerian women on domestic violence: evidence from 2003 Nigeria Demographic and Health Survey. African Journal of Reproductive Health, 9(2), 38-53. https://doi.org/10.2307/3583461

Prateek, S. Shrivastava, \& Saurabh R. Shrivastava. (2013). A Study of Spousal Domestic Violence in an Urban Slum of Mumbai. Int J Prev Med, 4(1), 27-32.

Rani, M., Bonu, S., \& Diop-Sidibe, N. (2004). An empirical investigation of attitudes towards wife-beating among men and women in seven sub-Saharan African countries. African Journal of Reproductive Health, 8, 116-136. https://doi.org/10.2307/3583398

Ritzer, G. (1996). Sociological theory. New York: McGraw Hill.

Srinivasan, S., \& Bedi, A. S. (2007). Domestic violence and dowry: Evidence from a South Indian village. World Development, 35, 857-880. https://doi.org/10.1016/j.worlddev.2006.08.005

UK Home Office. Domestic violence and abuse. (2013). Domestic abuse: guidance and support for the armed forces community and Domestic violence. Retrieved from https://www.gov.uk/guidance/domestic-violence-and-abuse

Umeora, O. U., Dimejesi, B. I., Ejikeme, B. N., \& Egwuatu, V. E. (2008). Pattern and determinants of domestic violence among prenatal clinic attendees in a referral centre, South-East Nigeria. J Obstet Gynaecol, 28, 769-774. https://doi.org/10.1080/01443610802463819 
United States Department of Justice, Office on Violence Against Women. (2017). Domestic Violence. Retrieved September 20, 2017, from https://www.justice.gov/ovw/domestic-violence

World Health Organisation. (2005). WHO Multi-county study on Women's Health and Domestic Violence against Women: Initial results on prevalence, health outcomes and women's responses. WHO, Geneva Switzerland.

World Health Organization. (2013). Global and regional estimates of violence against women: prevalence and health effects of intimate partner violence and non-partner sexual violence. Retrieved January 20, 2017, from http: www.who.int

Yusuf, N. (2000). Incidence and Dimension of Violence Against Women in the Nigerian Society. Centrepoint Journal.

\section{Copyrights}

Copyright for this article is retained by the author(s), with first publication rights granted to the journal.

This is an open-access article distributed under the terms and conditions of the Creative Commons Attribution license (http://creativecommons.org/licenses/by/4.0/). 